\title{
EL VECTOR DE FUERZA ARGUMENTATIVA：UNA HERRAMIENTA PARA MOSTRAR LA INFLUENCIA DE LAS METÁFORAS EN LA ARGUMENTACIÓN
}

\author{
The vector of argumentative force: a tool to show the influence of metaphors \\ in argumentation
}

Henry Campos Vargas ${ }^{1}$

A mi sobrina Ana Milagro

\begin{abstract}
RESUMEN
El presente artículo propone el concepto de vector de fuerza argumentativa como una herramienta útil para determinar el valor de una proposición dentro de una argumentación.
\end{abstract}

Palabras clave: argumento, falacias, metáfora, metáfora orientacional, fuerza de un argumento, fuerza argumentativa, vector.

\begin{abstract}
This paper proposes the concept of the vector of argumentative force to exam the value of a sentence in an argumentative sequence.
\end{abstract}

Key Words: argument, fallacies, metaphor, orientational metaphor, force of an argument, argumentative force, vector.

\footnotetext{
${ }^{1}$ Universidad de Costa Rica. Profesor de la Escuela de Filología, Lingüística y Literatura. Costa Rica.

Correo electrónico: hcamposv@yahoo.es

Recepción:29-02-17 Aceptación: 23-05-17
} 


\section{LAS METÁFORAS ORIENTACIONALES}

En un provocativo ensayo de opinión, el 5 de abril del 2015, Armando González, en su espacio del periódico La Nación titulado "Entre líneas", discutía a propósito de la participación ciudadana en relación con el sistema de fijación de precios de las tarifas de los autobuses:

Los transportistas ya anuncian oposición a la propuesta del MOPT, pero no delatan un interés específico y lanzan el ataque desde posiciones más elevadas, casi altruistas. Les inquieta "la restricción de la participación ciudadana" (2015, p. 19A).

Este texto muestra que:

(...) la función primera de las metáforas es cognitiva y ocupan un lugar central en nuestro sistema ordinario de pensamiento y lenguaje (Nubiola, 2000, p. 5).

En efecto, González establece la siguiente relación:

$$
\text { elevado }=\text { altruismo }
$$

El "Editorial" de ese mismo periódico, tres páginas antes, exaltaba la figura de tres de las más destacadas cabezas de la Iglesia Católica de finales del siglo XX y principios del XXI: Juan XXIII, Juan Pablo II y el papa Francisco:

Y, en este Domingo de Resurrección, debemos evocar a cada uno de ellos como indoblegables líderes espirituales cuyo paso por la Iglesia ha fortalecido la solidez de sus instituciones y la confianza y amor de los creyentes $\left(2015\right.$, p. $\left.18^{a}\right)$.

Dado que "indoblegable" equivale a aquello que no se dobla, es decir, lo que no se tiende hacia abajo, resulta que se presenta la imagen de tres líderes en posición vertical, donde:

$$
\text { erguido }=\text { valioso }
$$


Tal imagen es reforzada con el carácter espiritual de su liderazgo, ya que lo espiritual forma parte de una cadena de asociaciones mentales de la forma:

$$
\text { espiritual }=\text { racional }=\text { cabeza }=\text { arriba }
$$

No es casual que en esa misma página, una de las "Cartas a la columna” (espacio dedicado a quienes brevemente escriben críticas, comentarios o cartas públicas de opinión popular) destacara el triunfo de la Selección de Costa Rica en la Copa Mundial de Fútbol de Brasil 2014 al llegar a cuartos de final:

Esas voces son las mismas que rumbo al 2014 criticaron el empate con El Salvador en casa y contra Panamá, pero luego la vieron quedar octava en Brasil, derribando a los gigantes (Solano, 2015, p. 18A).

Aquí la metáfora "derribar gigantes” es más compleja, ya que involucra las relaciones "gigante-alto-arriba-más" y "derribar-suelo-abajo-menos", de lo que resulta que, quien derriba gigantes, pasa a ocupar la otrora posición elevada del derrotado.

Para Lakoff y Johnson (2001, p. 62) esto obedece a un sistema de pensamiento y de asociaciones primario, definido por cada cultura. Sin embargo, pese al carácter relativo que pudiera tener, presenta un entramado bastante consistente al interior de cada grupo humano.

Detrás de cada una de tales relaciones existe un sistema de valoraciones vinculados a una base experiencial, explican ambos autores: vivencias como la enfermedad y la muerte son la base para considerar que el suelo, la posición horizontal, la quietud, lo bajo, son menos y negativos; mientras que la posición de una persona sana, de pie, permite integrar conceptos como alto, vertical y crecimiento con lo positivo. Para ellos:

(...) parece que nuestros valores no son independientes, sino que deben formar un sistema coherente con los conceptos metafóricos de los que vivimos. No estamos afirmando que todos los valores culturales coherentes con un sistema metafórico existan en realidad, solamente que aquellos que existen y están profundamente establecidos son consistentes con el sistema metafórico (Lakoff, 2001, p. 60). 
Más adelante puntualizan:

En general, la elección de los valores a los que se concede prioridad es cosa de la subcultura en la que se vive, y parcialmente también de los valores personales. las diversas subculturas de una cultura principal comparten valores básicos, pero les conceden prioridades distintas (ibid).

Por esto, la "caída de los gobiernos" equivale a su término tal y como en la siguiente cita:

La estrategia de la dictadura en este asunto la ha llevado a la encrucijada en que la liberación de Leopoldo López es la caída de Nicolás Maduro y su régimen (Sánchez, 2015, p. 23 A).

Empero, en un contexto como el costarricense, se encuentra una cómica metáfora con una base experiencial diferente:

Pasado un tiempo nos cayó la peseta: estábamos en alitas de cucaracha (Kandler, 2015, p. 21A).

"Caer la peseta" es producto de la experiencia de hacer una llamada telefónica mediante la introducción de una moneda de veinticinco céntimos (una peseta) para pagar el servicio de telefonía. Así como la peseta activa el sistema, el "darse cuenta de una circunstancia" debe suscitar una reacción en el lector. En la cita, está presente un tipo de metáforas distinto, el "estar en alitas de cucaracha", es decir, en una situación delicada y peligrosa.

Diferente es el examen de la pertinencia o no de una obra de Henry Kissinger, o más concretamente, la crítica a que un canciller mostrara uno de los libros de este autor. En este contexto, Eduardo Ulibarri emplea distintas metáforas orientacionales. La mayoría de ellas es de carácter verbal:

En él aborda con profunda agudeza la relación entre su pasado milenario y sus decisiones (...) 
Entre ellos están el surgimiento de nuevas potencias (China, en prime lugar) (...)

Para afrontar con éxito estos retos y reconstruir un "nuevo orden" estable y asentado en normas (...)

Durante su exposición reflexionó sobre los retos y oportunidades del crecimiento (2015, p. 23 A).

“Abordar", "asentar", "crecer", "surgir" son todos verbos que denotan una acción hacia arriba, ya en su sentido conductal, ya en su etimología. Colofón a lo anterior es el empleo de otra metáfora para referirse a la edad del longevo político:

El pasado año, con 91 sobre sus espaldas, Kissinger publicó World Orden (“Orden Mundial") (ibid).

Bien cabe preguntarse sobre el valor que aportan las metáforas, en particular las orientacionales, a los procesos argumentativos. La respuesta parece compleja, empero, antes de contestarla debe considerarse el contexto en el que las metáforas se han desarrollados por un largo período de tiempo.

Por un lado,

In classical argumentation theory, metaphors usually lead to fallacies of reasoning: metaphors are governed by heuristic rules that never guarantee preservation of truth (Tindale y Fischer, citados por Ervas y Gola, 2016, p. 1).

En la teoría clásica de la argumentación, las metáforas usualmente conducen a falacias de razonamiento: las metáforas están gobernadas por reglas heurísticas que nunca garantizan la preservación de la verdad (traducción del autor).

Junto a esta situación, se tiene que:

Within argumentation theory, metaphor is traditionally viewed as a stylistic device that is used for ornamental purposes only (Wagemans, 2016, p. 80). 
Dentro de la teoría de la argumentación, la metáfora es vista tradicionalmente como un dispositivo estilístico que es usado únicamente con propósitos ornamentales. De esta manera, la valoración de las metáforas consistía en no aportar nada a los razonamientos o, incluso, invalidarlos; o estar reducidas a consideraciones meramente estéticas.

Sin embargo, desde el Tratado de la Argumentación de Perelman y OlbrechtsTyteca se ha defendido su valor en la argumentación. Estos autores desarrollaron una idea que tiene su origen en la Retórica de Aristóteles: al establecer el pensador de Estagira que la función de esta técnica era discernir los argumentos adecuados para cada contexto (1999, p. 1355b25,173), todo el aparato retórico, y en particular el ornamental, se subordinó al proceso argumentativo.

Desde esa época, el proceso se revitalización de la metáfora ha sido complejo, en palabras de Wagemans:

After 1980, when a number of publications caused a revolution in this field of study, metaphor came to be regarded as a conceptual device, a tool for thinking about one thing in terms of something else (...) A great number of studies showed how metaphor is important for understanding, reasoning, imagining, and communicating the same patterns emerging across different language, cultures and periods in comparable manifestations (2016, p. 79).

Después de 1980, cuando un número de publicaciones causó una revolución en este campo de estudio, la metáfora llegó a ser considerada como un dispositivo conceptual, una herramienta para pensar una cosa en términos de otra distinta (...) Un gran número de estudios ha mostrado cómo la metáfora es importante para comprender, razonar, imaginar y comunicar los mismos patrones que emergen a lo largo de los diferentes lenguajes, culturas y períodos en manifestaciones semejantes.

Para dicho autor, las principales vías de investigación en este campo se han desarrollado en dos sentidos: 
In studying the relation between metaphor and argument, some present-day scholars stick to the traditional conceptualization of metaphor as a stylistic device (e.g. GARSSEN 2009). This approach is premised on the idea that elements of argumentative discourse, if expressed in figurative language, can be transformed into literal language (Wagemans, 2016, p. 80).

Al estudiar la relación entre la metáfora y la argumentación, algunos académicos actualmente apuntan al concepto tradicional de la metáfora como un instrumento estilístico (por ejemplo GARSEEN 2009). Esta aproximación se basa en la idea de que los elementos del discurso argumentativo, si se expresan en un lenguaje figurativo, pueden ser transformados al lenguaje literal.

Por otro lado, en cambio:

Other scholars tend to view the relation between metaphor and argument along the same lines as Perelman and Olbrechts-Tyteca. Their approach is premised on the idea that metaphor is to be conceived in terms of argument schemes based on analogy, in which the concept of similarity plays a pivotal role (see e.g. Oswald \& Rihs, 2014, p. 141-143; Santibáñez, 2010, p. 947-978; Svačinová, 2014, p. 71-72) (Wagemans, 2016, p. 80).

Otros académicos tienden a ver la relación entre la metáfora y la argumentación en la misma línea que Perelman y Olbrechts-Tyteca. Su aproximación está basada en la idea de que la metáfora ha de ser concebida en términos de esquemas de argumentación basados en la analogía, donde el concepto de similaridad juega un papel fundamental (véase, por ejemplo, Oswald \& Rihs, 2014, p. 141-143; Santibáñez, 2010 p. 947-978; Svačinová, 2014, p. 71-72).

El propio Wagemans ofrece una nueva perspectiva de análisis materializada en sus Tablas de Periódicas de la Argumentación, que no serán objeto de examen en este trabajo, el cual, pretende contribuir a la discusión al ofrecer una vía adicional de estudio en este campo. 
Por ejemplo, para un fiel creyente del "socialismo bolivariano" la sola referencia a una "caída de Nicolás Maduro", tal y como se hizo líneas arriba, podría parecerle insultante, mientras que a un opositor le resultaría una imagen atractiva.

En un sentido semejante, un yihadista no aceptaría que se le presenten las figuras papales como "indoblegables líderes espirituales", mientras que para un ferviente católico le confirmaría su sistema de pensamiento.

Perelman y Olbrechts-Tyteca (1989, p. 274) llamaron a estos procesos figuras, ya de comunión, ya de elección, ya de presencia. A través de ellos, se logra una mayor empatía con el auditorio y, por lo tanto, la aceptabilidad de las tesis; se define con claridad el punto su vista o se reitera oportunamente aquello que se desea comprobar (1989). Sin embargo, lo anterior no muestra propiamente cuánto pueden incidir las metáforas, en este caso, orientacionales en los procesos argumentativos.

Para proponer una posible solución conviene partir de la constatación de un hecho: el corpus examinado es, básicamente, de opinión política, en sentido lato. Al ser de opinión y presentarse dentro del ámbito de la polis es factible identificar un espacio al que pertenecen: todos están relacionados con la toma de decisiones.

Las metáforas de todo tipo, y las orientacionales no son la excepción, se encuentran integradas en un complejo sistema de valores y valoraciones propios de una cultura que se transforman y evolucionan a lo largo del tiempo. Estos valores y, en especial las valoraciones a ellos asociadas, varían a lo largo del tiempo; y, tal y como Perelman y Olbrechts-Tyteca apuntan:

Los valores intervienen, en un momento dado, en todas las argumentaciones (1989, p. 132).

\section{EL VECTOR DE FUERZA ARGUMENTATIVA}

Van Dijk al estudiar la metáfora en el discurso ideológico apunta que:

(...) existen pocas figuras retóricas y semánticas tan persuasivas (2003, p. 120). 
Ahora bien, para tratar de identificar la forma como tales figuras retóricas intervienen en los procesos argumentativos, se precisa construir una herramienta adecuada.

En atención a que una argumentación es una especie de vector comunicacional, entendido como un acto de habla con una fuerza y dirección argumentativa específica en un contexto dado, el valor de un acto de argumentación podría representarse aproximadamente así:

$$
A_{t \rightarrow}(x)=\left[p \rightarrow(x) \cdot V_{t}(x)+R C L \rightarrow(x)\right]
$$

Aquí “A (x)” es el vector de fuerza argumentativa de la secuencia $x$ en el momento $t$, es definido como el resultado de la sumatoria de su validez lógica $R C L$ y el producto de la multiplicación de su grado de pertinencia $p$ por su valoración $V$ en una cultura dada. $p, V$ y $R C L$ tienen valores numéricos que, en los dos primeros casos, oscila entre 1 y 0 , mientras que en $R C L$ es 1 o 0 . Sin embargo, $V$ puede tener valor negativo.

El concepto de "fuerza argumentativa" es semejante, aunque no idéntico, al de "peso" empleado recientemente por Curtis K. J. Hyra, cuyo objeto de investigación se expresa en los siguientes términos:

I will answer the question of what it means for concepts, arguments, to have "weight" by examining our conceptions of weight in the physical world to see how the metaphor is used in situ (2016, p. 7).

Responderé la pregunta sobre qué significa para conceptos, argumentos, tener "peso" mediante el examen de nuestras concepciones de "peso" en el mundo físico para ver cómo la metáfora es empleada en este campo.

Efectivamente, la expresión “este es un argumento de peso" es una metáfora física. Su empleo generalizado en muchos contextos, científicos y cotidianos, lleva a que en el estudio se postule: 
I argue that weight is deserving of being adopted by the rhetorical cannon on its own (2016, p. 12).

Yo arguyo que el "peso" merece ser adoptado por el canon retórico por sí mismo.

Sin embargo, a diferencia del estudio supra citado, aquí se propone un modelo cuantitativo que podría demostrar lo que se ha venido afirmando: que el empleo de las metáforas no es indiferente en contextos argumentativos. Modelos cuantitativos también han sido propuestos por distintos investigadores respecto de otros conceptos, tal es el caso de la noción de "robustez argumentativa" que aquí no será considerada (entre otros puede consultarse Véliz, 1988 y van Eemeren, et al., 2006).

En una argumentación en general, el hablante debe llevar a cabo una serie de secuencias de habla con una fuerza argumentativa cuya suma total debe aspirar a superar el que aquí se llama umbral de persuasión. Este consiste en un valor numérico que en cada sujeto está asociado al nivel en el que considera se le ha convencido de una determinada tesis luego de una experiencia argumentativa.

Nótese que la "dirección argumentativa" viene determinada por la pertinencia o no del enunciado $x$, así como por su validez; de ambos sumandos, el primero tiene la particularidad de indicar de manera más directa una dirección a favor, en contra o de desvío respecto de la "meta" persuasiva y un valor numérico asignado a dicha pertinencia; ya que, el segundo, o carece de valor (cuando su resultado es 0) o fortalece la argumentación.

La inserción de la argumentación $x$ en un sistema de valores, no podía ser de otro modo, ya que las reglas del cálculo lógico son considerablemente estables y comunes a cualquier tipo de argumentación, empero, no brindan un criterio que permita dar preferencia a un argumento frente a otro. Es por esto que la dirección requerida por este vector debe tener su origen en otro de los componentes del modelo. Las metáforas, entre ellas las orientacionales, tienen aquí su contribución en la argumentación.

Desde el punto de vista de la lógica, $R C L$ solo arrojaría en sistemas de dos valores (falso y verdadero) pues la argumentación es válida o inválida. A la validez se le puede asignar un valor número específico, por ejemplo, 1, mientras que a la invalidez, 0 . Esto es 
consistente con la experiencia empírica: los argumentos inválidos pueden tener mucha fuerza argumentativa en función de su valoración no lógica.

Si el vector de fuerza argumentativa fuera el resultado de una multiplicación, la experiencia desmentiría el modelo propuesto, ya que ofrecería un vector de fuerza argumentativa con valor 0 , mientras que la cotidianidad manifiesta que puede llegar a tener un valor mayor. Tal es el caso de la poderosa influencia de numerosas falacias: si bien su valor lógico puede ser 0 , el juicio correspondiente al otro sumando cambia su ponderación total.

En igual sentido, argumentaciones equivocadas o contraproducentes, pueden arrojar un valor negativo para el vector, lo que equivale a un retroceso o debilitamiento del proceso de persuasión.

Es claro que $V$ es a su vez la función de una gama de valoraciones, por ejemplo, estéticas, literarias, morales, pragmáticas, económicas. De este juego participan las metáforas orientacionales. Debe tenerse presente que mientras mayor sea el número de ámbitos considerados, más precisa será su ponderación. Para determinar el valor que habrá de darse en cada caso, puede recurrirse a estudios estadísticos.

Por su parte, $p$ representa la pertinencia del enunciado respecto de la argumentación y tiene un valor entre 0 y 1 , donde este último representa un grado de oportunidad total para el enunciado. Piénsese, por ejemplo, en el valor argumentativo de citar una estrofa del Himno Nacional de un país. A propósito de una discusión sobre la eficiencia de un motor, su pertinencia es poca. Sin embargo, ante una amenaza bélica su pertinencia es total.

Tal y como se aprecia, $p$ es una especie de corrector que previene contra prácticas en las que únicamente se aglutinan afirmaciones sin conexión entre sí. De acuerdo con el modelo, la ausencia de $p$ conllevaría que fácilmente se atravesara el umbral de persuasión mediante el simple recurso de "escribir" y "escribir".

$p$, igualmente, muestra por qué secuencias descriptivas, históricas, simples valoraciones, pueden aumentar el valor del vector $A$ en la argumentación en general. Todas 
estas tienen un valor $R C L$ de 0 , ya que no son argumentos; no obstante, tienen importancia ("peso", si se quiere) en el complejo proceso de la argumentación.

Las metáforas orientacionales participan de estas características: desde un punto de vista estrictamente lógico, se solía afirmar que todas las metáforas eran "falsas". Decirle a alguien que "me lleva muy alto", a menos que se vaya en avión o un elevador..., es estrictamente falso.

Aunque esto sea cierto en algunos casos, Lakoff y Johnson (2001, p. 42) han mostrado que esto no es del todo correcto, dado que las metáforas forman parte de la forma de ver el mundo y de los complejos procesos de configuración del sentido de las percepciones y los procesos comunicativos. No obstante, muchas de tales metáforas pueden presentar también un valor 0 en $R C L$, pese a contribuir sobradamente en una argumentación.

Adicionalmente, $p$ confirma la importancia de recursos como la repetición de un mensaje a lo largo de un discurso, ya que, de alguna manera, refuerzan el proceso de persuasión (lo que es ampliamente aprovechado en áreas como la publicidad comercial y política).

Ahora bien, ¿por qué no es posible apreciar esto en la lógica clásica -representada aquí de manera aproximada en $R C L$ ? La respuesta podría ser que, sencillamente, la lógica clásica se aplica en contextos donde todas las anteriores variables del modelo se asumen como constantes, lo que hace irrelevante su ponderación, con la posible asignación de un valor de 0 a $V$. Esto es consistente nuevamente con la experiencia, toda vez que la lógica clásica se ocupa de validez, no de conceptos como el de fuerza argumentativa.

En efecto, definido un contexto de discusión, por ejemplo, resolver un problema matemático, se aplica el principio ceteris paribus, lo que permite concentrarse únicamente en las reglas del cálculo por aplicar: el tiempo no sufre alteraciones y las valoraciones se consideran estáticas.

Algo semejante ocurre en la discusión judicial, cuando lo que interesa es demostrar la culpabilidad o no de un imputado: el marco de referencia está bastante definido y las 
variables son pocas. La discusión se concentra en el examen de la prueba, su valoración y la ley.

Como acto de habla complejo que es la argumentación, el valor total del vector de fuerza argumentativa podría representarse de la siguiente forma:

$$
\Sigma_{1}{ }^{n} A_{t} \rightarrow\left(x_{n}\right)=\left[p \rightarrow\left(x_{n}\right) \cdot V_{t}\left(x_{n}\right)+R C L \rightarrow\left(x_{n}\right)\right]
$$

Es decir, es la sumatoria del valor de fuerza argumentativa de cada uno de sus enunciados. Esto, nuevamente, es consistente con la experiencia, por cuanto secuencias argumentativas "malas" afectarán la fuerza argumentativa total, mientras que las "buenas" aumentarán su valor.

\section{ANÁLISIS DE UN CASO PARTICULAR}

El examen de un vector de argumentación puede ilustrarse, desde el punto de vista de las metáforas orientacionales, con el análisis de unos pasajes del Editorial del diario La Nación del 26 de abril del 2015, titulado Mercaderes de la muerte, donde se expone la situación de los refugiados en Europa (se prescinde de la definición del umbral de persuasión):

La trágica situación de los inmigrantes africanos, que cada día desafían las aguas del Mediterráneo en busca de trabajo y mejores condiciones de vida en Europa, desborda el retrato que suelen describir algunos medios de comunicación del continente. Y, cada día también, las cifras de las víctimas de los percances fatales en el mar crecen en forma alarmante (p. 26A).

Dos metáforas orientacionales se ofrecen en la cita: desborda y crecen, las que forman parte de sendas afirmaciones a las que se les aúnan determinados juicios de valor:

$$
\mathrm{A}_{\mathrm{t}} \rightarrow(\mathrm{x})=\left[\mathrm{p} \rightarrow(\mathrm{x}) \cdot \mathrm{V}_{\mathrm{t}}(\mathrm{x})+\mathrm{RCL} \rightarrow(\mathrm{x})\right]
$$

En ambos casos, no se emplean en razonamientos formales, por lo que su valor $R C L$ es 0. En atención a que el Editorial pretende crear conciencia sobre la magnitud de la crisis, la pertinencia de su empleo es total, lo que se representará con el valor numérico 1. 
Respecto de su valoración, ambas metáforas están correctamente construidas y son coherentes con el discurso. Sin embargo, desborda merece una valoración mayor, dado que transmite la imagen al lector de la navegación, cuando es la travesía por el mar la que constituye uno de los mayores problemas de la situación. En razón de lo anterior, a desborda se le asigna un 1 y a crecen 0.5 .

El ejercicio anterior pone de manifiesto que ambas metáforas contribuyen y refuerzan el proceso argumentativo. Tal efecto se confirma en esta otra cita, en la que también se identifican metáforas orientacionales:

Precisamente, a este sentimiento respondió la convocatoria a una cita de alto nivel de la Unión Europea, llevada a cabo el jueves en Bruselas, a las cual asistieron gobernantes y diplomáticos de los países miembros. En ella se acordó incrementar el ámbito de las acciones de salvamento y triplicar los presupuestos correspondientes (ibid).

"Alto nivel" e "incrementar" (del latín increscere) son las dos nuevas metáforas orientacionales involucradas. Ambas forman parte de meras secuencias descriptivas de actividades, por lo que no comprometen las nociones de validez o, su opuesto, la invalidez. La referencia de la "cita de alto nivel" es pertinente, a la vez que contribuye a dimensionar la importancia de la intervención política, lo que arroja una calificación $p . V$ de 1 . En cuanto a "incrementar" su pertinencia es razonable (0.75), pero su valor no lo es tanto $(0.5)$, lo que resulta en un 0.375 .

Ahora bien, independientemente del cuál sea el valor asignado al umbral de persuasión, es evidente que estas metáforas orientacionales han contribuido a aproximar al editor al éxito en su argumentación.

\section{CONCLUSIONES}

El examen del corpus de metáforas orientacionales permitió identificar la necesidad de construir un modelo apropiado que mostrara su incidencia en los procesos argumentativos. Con este propósito, se creó el concepto de fuerza argumentativa de un enunciado. 
La definición de este concepto ha mostrado ser una herramienta consistente para el examen de dinámicas argumentativas. En particular, ha sido coherente con la influencia de falacias, la introducción de enunciados inatingentes y el empleo de metáforas orientacionales. Además, hay indicios que apuntan a que igualmente permite ponderar la incidencia de otro tipo de metáforas y figuras retóricas en la argumentación en general.

Merced a su empleo, pudo comprobarse que las metáforas orientacionales, cuando son bien elaboradas y son pertinentes, carecen de poco impacto lógico, pero refuerzan la argumentación en procura de la persuasión.

\section{BIBLIOGRAFÍA}

Aristóteles. (1990). Retórica. (2ª reimpresión). Madrid, España: Editorial Gredos, S. A.

Ervas, Francesca y Gola, Elisabetta. (2016). “Arguing by metaphors". En Rivista Italiana di Filosofia del Linguaggio. Metaphor in Language and Argumentation, X (2), 1-6. Recuperado de http://www.rifl.unical.it/index.php/rifl (Consultado el $1^{\circ}$ de mayo del 2017).

González, Armando. (2015). “Editorial: Fortaleza y esperanza”. En La Nación. 05 de abril, 18A.

González, Armando. (2015). "Editorial: Mercaderes de la muerte”. En La Nación. 26 de abril, 26A.

Kandler Sancho, Ricardo. (2015). “El mundo de Kandler”. En La Nación. 05 de abril. 21 A.

Lakoff, George y Johnson, Mark. (2001). Metáforas de la vida cotidiana. Traducción de Carmen González Marín. Madrid, España: Ediciones Cátedra, (Grupo Anaya, S. A.).

Perelman, Ch. y Olbrechts-Tyteca, L. (1989). Tratado de la argumentación. La nueva retórica. Traducción española de Julia Sevilla Muñoz. (1ª reimpresión). Madrid, España: Editorial Gredos, S. A. 
Sánchez, Carlos. (2015). “Liberar a Leopoldo es la caída de Maduro”. En La Nación. 05 de abril, 23A.

Solano, Johel. (2015). “Hipocresía en la Selección”. En La Nación. 05 de abril. p. 180A.

Ulibarri, Eduardo. (2015). “Leer a Henry Kissinger”. En La Nación. 05 de abril, 23A.

van Dijk, Teun A. (2003). Ideología y discurso. (1ª ed.). Barcelona, España: Editorial Ariel, S. A.

van Eemeren, Frans H. y Grootendorst, Rob. (2002). Argumentación, comunicación y falacias. Una perspectiva pragma-dialéctica. (2a ed.). Santiago, Chile: Ediciones Universidad Católica de Chile.

van Eemeren, F. H., Grootendorst, R., y Snoeck Henkemans, F. (2006) Argumentación: análisis, evaluación, presentación. Buenos Aires, Argentina: Biblos.

Véliz, Mónica. (1988). "Evaluación de la madurez sintáctica en el discurso escrito", RLA. Revista de Lingüista Teórica y Aplicada, (26). 105-141.

Wagemans, Jean H. M. (2016). "Analyzing metaphors in argumentative discourse". Rivista Italiana di Filosofia del Linguaggio. Metaphor in Language and Argumentation, X (2), 79-94. Recuperado de http://www.rifl.unical.it/index.php/rifl (Consultado el $1^{\text {o }}$ de noviembre de 2016).

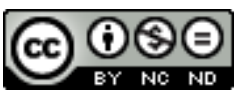

\footnotetext{
Esta obra está bajo una licencia de Creative Commons Reconocimiento-NoComercialSinObraDerivada 4.0 Internacional
} 